
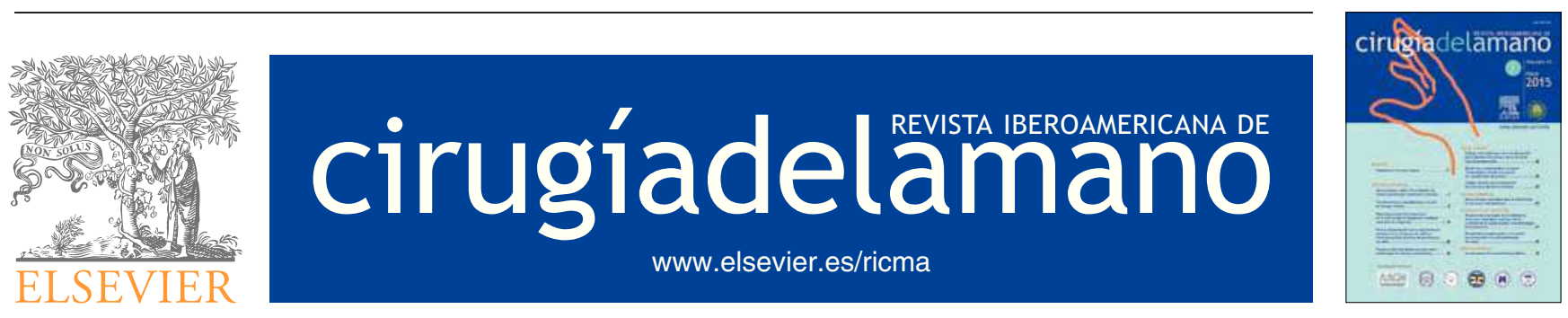

ARTÍCULO ORIGINAL

\title{
Resultado precoz del tratamiento de la enfermedad de Dupuytren mediante inyección de colagenasa
}

\section{R.A. Pérez-Giner* y L. Aguilella}

Servicio de Cirugía Ortopédica y Traumatología, Hospital Universitario de La Ribera, Alzira, Valencia, España

Recibido el 5 de septiembre de 2014. Aceptado el 21 de febrero de 2015.

\section{PALABRAS CLAVE \\ Enfermedad de \\ Dupuytren; \\ Tratamiento; \\ Colagenasa}

\section{KEYWORDS}

Dupuytren disease;

Treatment;

Collagenase

\begin{abstract}
Resumen
Objetivo: Presentar nuestro protocolo de tratamiento y el resultado precoz obtenido en una serie inicial de pacientes tratados con colagenasa.

Material y método: Estudio prospectivo sobre 40 pacientes afectos de enfermedad de Dupuytren, tratados mediante colagenasa en 47 dedos. Los criterios de inclusión han sido cuerdas palpables con una flexión metacarpo-falángica y/o interfalángica proximal superior a $20^{\circ}$. Se han recogido los grados de contractura de las articulaciones metacarpo-falángica, interfalángica proximal e interfalángica proximal con metacarpo-falángica en máxima flexión, antes y después del tratamiento al mes y a los tres meses de la inyección.

Resultados: Se ha obtenido extensión completa (contractura igual o menor de $5^{\circ}$ ) en el $53 \%$ de los dedos tratados, con una media de 1,19 inyecciones por dedo. El 91\% de los pacientes ha considerado satisfactorio el resultado. La corrección en las metacarpo-falángicas ha sido completa en el $71 \%$ y en las interfalángicas proximales en el $43 \%$. Cuando la interfalángica proximal se corregía previamente con la metacarpo-falángica en flexión, su extensión completa se ha obtenido en el $77 \%$. Se detallan las características de las roturas cutáneas observadas.

Conclusión: La inyección de colagenasa es un método efectivo de tratamiento, con una tasa elevada de satisfacción en los pacientes.

๑ 2015, SECMA. Publicado por Elsevier España, S.L.U. Este es un artículo Open Acces distribuido bajo los términos de la licencia CC BY-NC-ND (http://creativecommons.org/licenses/by-nc-nd/4.0/).
\end{abstract}

Primary results of treatment of Dupuytren disease with collagenase injections

\begin{abstract}
Objective: To describe our treatment protocol and the early outcomes of a case series of patients treated with collagenase.

Material and method: A prospective study was conducted on 40 patients with Dupuytren's disease, treated by injecting collagenase into 47 fingers. Inclusion criteria were palpable cords and
\end{abstract}

*Autor para correspondencia.

Correo electrónico: perezginerrosana@gmail.com (R.A. Pérez Giner).

( $)$ 2015, SECMA. Publicado por Elsevier España, S.L.U. Este es un artículo Open Acces distribuido bajo los términos de la licencia CC BY-NC-ND (http://creativecommons.org/licenses/by-nc-nd/4.0/). 
a flexion contracture of the metacarpophalangeal or proximal interphalangeal joint of $20^{\circ}$ or more. Patient data was recorded before injection and during follow-up visits at one month and three months.

Results: Reduction in contracture to normal (between $0^{\circ}$ and $5^{\circ}$ ) was obtained in $53 \%$ of fingers treated. The mean number of injections was 1.19 . Satisfaction was recorded in $91 \%$ of patients. Full extension was achieved in $71 \%$ of metacarpophalangeal joints, and in $43 \%$ of proximal interphalangeal joints. When the interphalangeal joint could be passively corrected with the metacarpophalangeal joint in maximal flexion, full extension was achieved in the former joint in $77 \%$ of cases. Skin laceration characteristics have been detailed.

Conclusion: Collagenase injection is an effective treatment for Dupuytren's disease, with a high level of patient satisfaction.

(c) 2015, SECMA. Published by Elsevier España, S.L.U. This is an open access article under the CC BY-NC-ND license (http://creativecommons.org/licenses/by-nc-nd/4.0/).

\section{Introducción}

La enfermedad de Dupuytren es un trastorno fibroproliferativo progresivo de la aponeurosis palmar, caracterizado por el desarrollo inicial de nódulos y posteriormente de cuerdas, capaz de producir contracturas en flexión de las articulaciones digitales. La severidad de estas contracturas es variable, al igual que la velocidad de progresión de la enfermedad. Es una patología más frecuente en los varones y suele afectar preferentemente al cuarto y quinto dedo ${ }^{1}$. Tradicionalmente la enfermedad de Dupuytren se ha venido tratando con variadas técnicas quirúrgicas: fasciectomía selectiva, dermofasciectomía, fasciotomía percutánea, etc., habiéndose obtenido unos resultados habitualmente satisfactorios ${ }^{2}$. Los principales problemas del tratamiento quirúrgico son la elevada tasa de recidiva de la enfermedad y su morbilidad ${ }^{3}$.

Desde hace años se ha venido investigando la posibilidad de efectuar un tratamiento no quirúrgico, mediante la inyección de diversas sustancias en las cuerdas de la enfermedad de Dupuytren. Entre estas, el tratamiento enzimático con la colagenasa del Clostridium Histolyticum ha demostrado ser eficaz y seguro en varios ensayos clínicos ${ }^{4-7}$. Desde su aprobación en el 2010 por la Food and Drug Administration (FDA) y en el 2011 por la European Medicine Agency (EMA), este nuevo tratamiento farmacológico ha experimentado una notable difusión y ha sido implantado en numerosos centros.

El objetivo del presente estudio es analizar la eficacia a corto plazo de este tratamiento y evaluar las complicaciones observadas en nuestros pacientes tratados con colagenasa. Se describe asimismo el protocolo de tratamiento utilizado y el algoritmo de manejo de los pacientes una vez finalizados los tres meses de seguimiento.

\section{Material y método}

Se ha efectuado un estudio prospectivo observacional de una serie de 40 pacientes afectos de enfermedad de Dupuytren, tratados mediante inyección de colagenasa del Clostridium Histolyticum en $\mathbf{4 7}$ dedos, que han acudido a las consultas externas del Servicio de Cirugía Ortopédica y Traumatología del Hospital Universitario de La Ribera desde octubre del 2011 a marzo del 2014. Este periodo ha incluido el aprendizaje de la técnica.
El criterio de inclusión ha sido la presencia de una cuerda palpable en cualquier dedo, primaria o recidivada, con la excepción del pulgar ${ }^{4,8}$, causante de una contractura de la articulación metacarpofalángica (MCF) y/o interfalángica proximal (IFP) igual o mayor de $20^{\circ}$. Todos los pacientes han dado su consentimiento informado para el tratamiento y específicamente para el estudio, que ha sido previamente aprobado por el Comité Ético de Investigación Clínica del hospital. El tratamiento se ha efectuado en la zona de consultas externas del hospital, tomando medidas de antisepsia tanto para la punción como para el estiramiento, y se han utilizado las dosis recomendadas en la ficha técnica del medicamento para cada articulación.

La elección del punto de inyección, aspecto muy relevante en pacientes que presentan afectación en dos articulaciones, se ha realizado en función de las características de las cuerdas del dedo afecto. En aquellos pacientes donde solo existía afectación de una articulación, MCF o IFP, la inyección se ha efectuado directamente sobre la cuerda responsable de la contractura. Sin embargo, cuando existía afectación de dos articulaciones, para obtener el mayor rendimiento clínico con una única punción, se ha utilizado la maniobra de hiperflexión MCF con objeto de determinar la cuerda que se iba a inyectar. Al efectuar esta maniobra (fig. 1), si mejoraba sustancialmente la contractura de IFP, demostrando por tanto la dependencia de la contractura de la cuerda pretendinosa, la inyección se ha efectuado en dicha cuerda. En el caso de no observar reducción en la contractura de IFP con la maniobra de hiperflexión MCF, se demuestra la dependencia de la contractura de una cuerda central o lateral y se ha optado por efectuar la inyección en aquella articulación más afectada, advirtiendo al paciente de la previsible conveniencia de una segunda inyección para tratar la otra articulación. La dosis total de colagenasa para cada caso se ha administrado fraccionada en tres puntos contiguos de la misma cuerda.

El estiramiento de la cuerda se ha realizado, en esta serie, a las 24 horas de la administración de colagenasa y siempre bajo anestesia local, a diferencia de la punción, para la que no se recomienda su uso. En primer lugar, se le ha pedido al paciente que estire activamente el dedo tratado. Con esta maniobra se produce en algunos casos la rotura completa o parcial de la cuerda. A continuación se ha efectuado la manipulación pasiva del dedo siguiendo el protocolo de estirar primero la articulación no tratada, mientras se mantiene la 

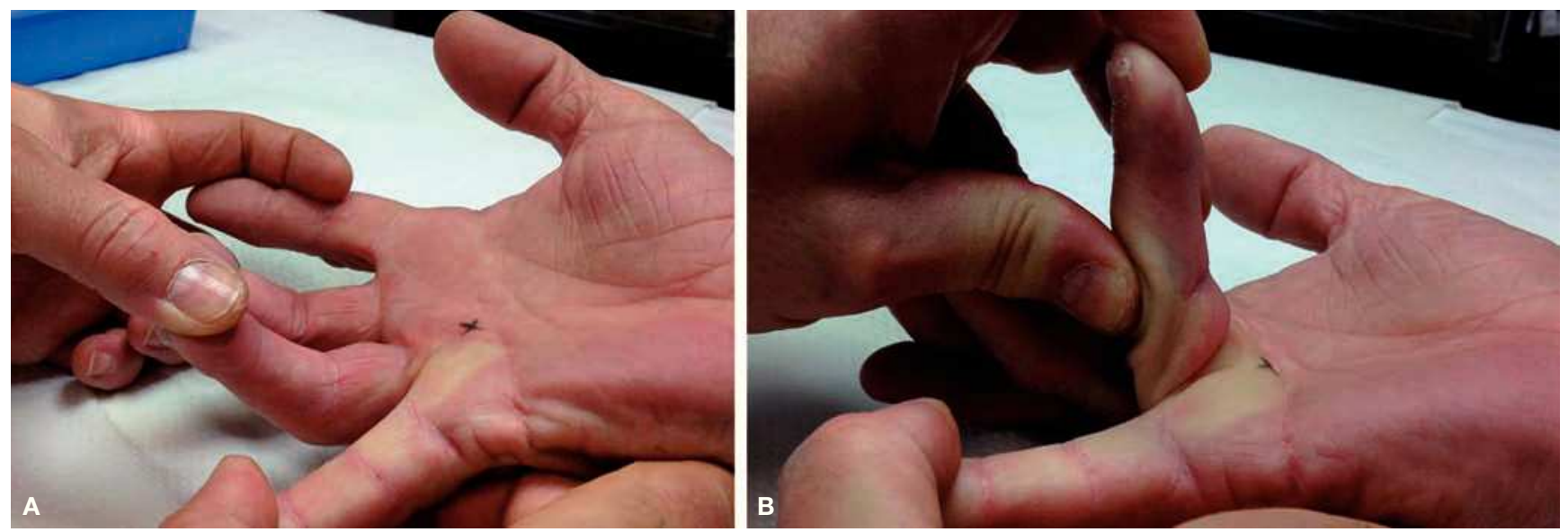

Figura 1 Cuarto dedo de la mano derecha afecto de la enfermedad de Dupuytren, con implicación de las articulaciones MCF e IFP (A). Presenta corrección de la contractura de IFP con la flexión máxima de la MCF (B).

tratada en flexión. Posteriormente se estira la articulación tratada manteniendo la otra en flexión. Finalmente, se estiran ambas articulaciones de forma conjunta. Estas maniobras se han repetido hasta tres veces, con intervalos de descanso, antes de considerar ineficaz el tratamiento.

En todos los pacientes se ha recogido previamente al tratamiento una serie de datos epidemiológicos, el tipo de cuerdas presentes y los grados de contractura de cada articulación, en el caso de IFP con y sin flexión MCF. Antes del estiramiento se han registrado los efectos secundarios de la punción y la utilización de medicación durante las primeras 24 horas. Tras el estiramiento se ha prestado especial atención a la presencia de desgarros cutáneos, que se han cuantificado en $\mathrm{mm}$. Se ha insistido a los pacientes en la importancia de utilizar una férula nocturna durante los primeros tres meses, así como en la realización de ejercicios activos de extensión a partir del día siguiente del estiramiento.

Todos los pacientes han sido revisados en consulta a la semana, al mes y a los tres meses, repitiendo en todas las visitas las mediciones de las contracturas articulares. Los pacientes con desgarros cutáneos extensos han sido revisados con mayor frecuencia hasta su cicatrización completa. En la última revisión se ha valorado la satisfacción del paciente mediante una escala numérica, donde el valor 1 corresponde a "nada satisfecho" y el valor 10 a "completamente satisfecho".

Al finalizar el seguimiento a los tres meses de evolución, cada paciente ha sido evaluado mediante un algoritmo de toma de decisiones (fig. 2). Se ha considerado un tratamiento objetivamente efectivo si la contractura residual ha sido menor o igual a $5^{\circ}$, en cuyo caso se ha dado el alta al paciente o se le ha propuesto el tratamiento de otro dedo si tenía más radios afectos. En aquellos casos donde el resultado no ha sido la extensión completa, se ha valorado la satisfacción subjetiva del paciente. Los pacientes han optado entonces por una segunda inyección o, si han considerado suficiente la mejoría obtenida, han sido dados de alta. En algunos casos, por las características de la contractura (por ejemplo, una contractura asociada de IFP y MCF que apenas se ha modificado) o por falta de colaboración del paciente

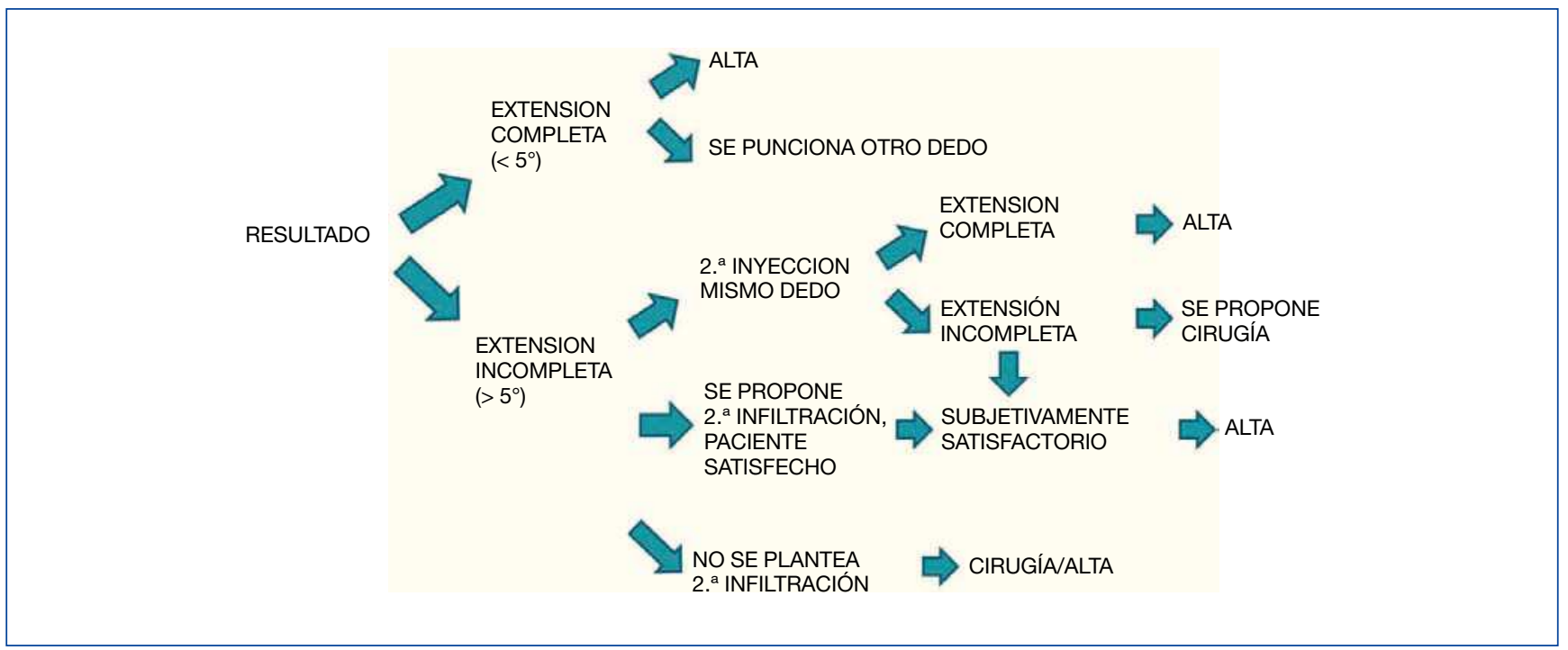

Figura 2 Algoritmo de toma de decisión terapéutica a los tres meses de evolución. 
(no seguir las indicaciones recibidas tras el tratamiento), no se ha considerado oportuno plantear una segunda inyección, pero sí se les ha ofrecido la posibilidad de cirugía. Aquellos pacientes que sí han recibido una segunda inyección siguen el mismo protocolo que en la primera. Tras la segunda inyección, si no se obtiene un resultado subjetivamente satisfactorio, se indica el tratamiento quirúrgico.

\section{Resultados}

Los 40 pacientes incluidos en el estudio tenían una edad media de 66 años (rango 52-82), con predominio de varones (96\%). Han sido tratados 47 dedos, el quinto en 23 casos (49\%), el cuarto en 20 casos (43\%), el tercero en 3 casos (6\%) y afectación de tercero y cuarto por una misma cuerda en 1 caso (2\%). La mano afecta ha sido la izquierda en 29 casos y la derecha en 18.

Los pacientes presentaban en su mayoría cuerdas pretendinosas asociadas a centrales $(43 \%)$, seguidas de cuerdas pretendinosas aisladas (30\%) y de cuerdas laterales cubitales $(9 \%)$. Se han observado variadas asociaciones entre cuerdas pretendinosas, centrales y/o laterales. Las punciones iniciales se han llevado a cabo en cuerdas pretendinosas en 37 casos, y en cuerdas centrales o laterales en 10 casos. Nueve pacientes han requerido una segunda inyección, con mayor frecuencia debido a cuerdas laterales únicas $(33,3 \%)$. El resultado clínico obtenido a los tres meses ha sido la extensión completa en 25 casos (53\%). Si añadimos los pacientes sin extensión completa pero satisfechos con resultado y que no desean más tratamiento, podemos considerar satisfactorios 43 casos (91\%). Se han efectuado 56 inyecciones hasta considerar finalizado el tratamiento, siendo la media de 1,19 inyecciones por dedo tratado.

La articulación MCF ha ofrecido mejores resultados (extensión completa en el 71\%) que la IFP (43\%). El subgrupo de articulaciones IFP que mejoraban con la flexión MCF ha obtenido extensión completa en el 77\%. Los grados de contractura media de cada articulación al inicio, al mes y los tres meses se recogen en la figura 3. La mejora media de la contractura ha sido del $85 \%$ en las MCF, del $73 \%$ en las IFP y del $80 \%$ en el subgrupo de IFP que mejoraban con flexión MCF.

Si se analizan los casos que no han alcanzado la extensión completa, en la MCF son 11 , presentado 5 casos $10^{\circ}$ de contractura residual, 3 casos $15^{\circ}$, un caso $20^{\circ}$ (con una mejoría de contractura de $55,5 \%$ ), otro $40^{\circ}$ (mejoría del $43 \%$ ) y un último caso con ausencia de corrección. En las IFP, 17 casos no han conseguido extensión completa, de los cuales 12 presentan $<20^{\circ}$ de contractura final. En el grupo de IFP que mejora con la maniobra de hiperflexión de la MCF, únicamente 3 de los 13 casos no han alcanzado extensión completa, pero con una mejoría de la contractura $>80 \%$.

Los efectos secundarios más habituales han sido locales en forma de tumefacción y hematoma. También ha sido frecuente que los pacientes precisaran algún tipo de analgesia durante las primeras 24 horas tras la punción de colagenasa (36\% de los casos). En cinco casos se ha observado linfangitis. La complicación más importante del tratamiento ha sido la rotura cutánea, que se ha producido en el $48 \%$ de los casos. Estas roturas se han clasificado en función de su tamaño (fig. 4). A pesar del aspecto llamativo en cuatro casos (tres de $1 \mathrm{~cm}$ y uno de $5 \mathrm{~cm}$ ), todas han tenido una evolución satisfactoria, solucionándose mediante curas periódicas en consultas externas. En algunos pacientes se ha observado la aparición de flictenas hemorrágicas antes o durante las maniobras de estiramiento, siendo estas un signo de rotura inminente de la piel. Para minimizar el desgarro cutáneo en estos casos, hemos encontrado útil efectuar una pequeña incisión longitudinal de drenaje de la flictena, que disminuye la tensión de la piel (fig. 5).

La satisfacción global del paciente al final del tratamiento, valorada mediante una escala numérica de 1 a 10 a los tres meses de evolución, ha sido de 8,8 de media (rango 5-10).

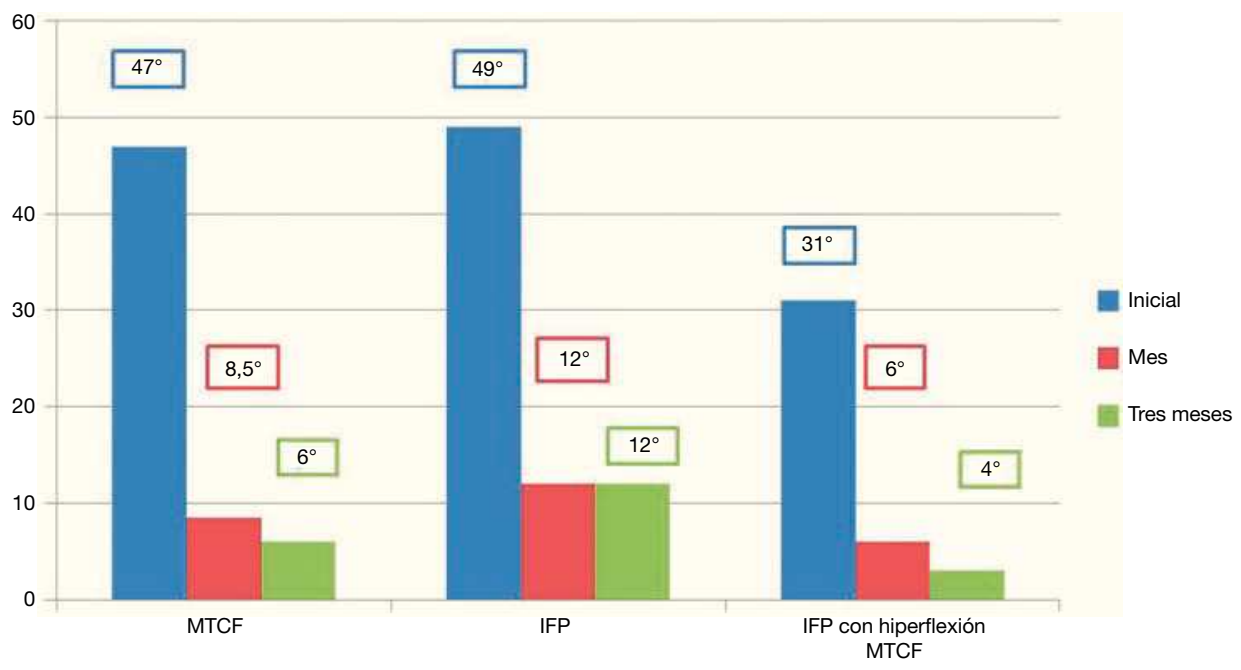

Figura 3 Grados de contractura articular, antes de la inyección de colagenasas, al mes y a los tres meses. 


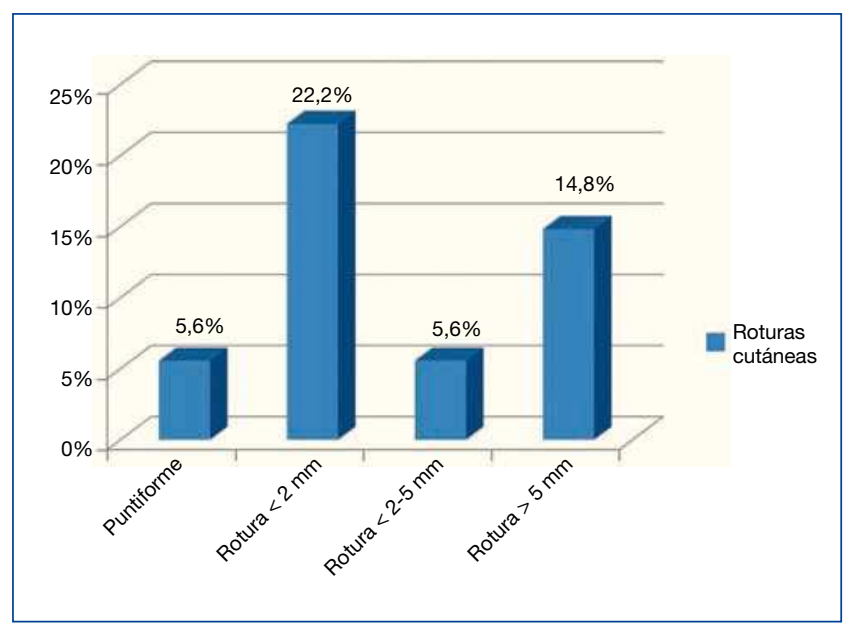

Figura 4 Descripción de las roturas cutáneas según su tamaño.

\section{Discusión}

El uso de la colagenasa en la enfermedad de Dupuytren se ha establecido como una alternativa eficaz y segura frente al tratamiento quirúrgico tradicional. Tras los conocidos ensayos clínicos CORD I y CORD II que precedieron a su aprobación $\mathrm{n}^{4-6}$, otros estudios multicéntricos han continuado comunicando resultados satisfactorios, con ausencia de efectos secundarios graves ${ }^{7,9}$.

Los resultados obtenidos en nuestros pacientes confirman lo observado por otros autores ${ }^{3,6,9,10}$, respecto a la mejor respuesta de las articulaciones MCF en comparación con las IFP (tabla 1). Los estudios más extensos hasta la actualidad son los denominados JOINT-I (Estados Unidos) y JOINT-II (Australia y Europa) ${ }^{7}$, que incluyen un total de 879 articulaciones afectas en 587 pacientes, con una media de inyecciones de $1,2 \pm 0,5$ por cuerda, siendo la articulación MCF $(60 \%$ de casos con extensión completa) la que mejores resultados presenta respecto a la IFP (32\%). Es destacable que la mayoría de los pacientes han referido un resultado muy satisfactorio $(71 \%)$ o bastante satisfactorio (21\%). En nuestro entorno, Martín Ferrero ${ }^{3}$ obtiene similares resultados, con una reducción de $64^{\circ}$ a $4^{\circ}$ en las MCF y de $83^{\circ}$ a $15^{\circ}$ en la IFP. En un reciente estudio multicéntrico ${ }^{12}$ se ha obtenido extensión completa de la MCF en el $80 \%$ de los casos, manteniéndose esta durante el primer año de evolución; en cambio las IFP, que presentan un $66 \%$ de extensión completa al mes, han sufrido un deterioro progresivo en este mismo periodo.

Es variable la media de inyecciones utilizadas en cada cuerda ${ }^{9}$, dependiendo de cómo se defina el éxito del tratamiento. Si este se considera como la obtención de la extensión completa, obviamente serán más numerosas las punciones que si consideramos la satisfacción del paciente como criterio de éxito. Aunque no parece haber contraindicación para efectuar repetidas inyecciones ${ }^{13}$, la técnica pierde entonces su relación coste-efectividad ${ }^{14-16}$. En nuestra serie, con una media de 1,19 inyecciones por dedo, consideramos que la técnica ha tenido un coste razonable.

Puede ser cuestionable el tratamiento con colagenasa en pacientes en los que existe afectación de varios dedos de la misma mano, o en los que la severidad de las contracturas hace prever tres o más inyecciones. El motivo es doble: la prolongación en el tiempo del tratamiento, debido a la autorización vigente de usar una única dosis cada vez, y el elevado coste en estos casos. Los resultados del reciente ensayo clínico multicéntrico abierto en fase $\mathrm{Illb}^{17}$, en el que se han aplicado dos dosis completas simultáneas de colagenasa a cada paciente, tanto para las articulaciones MCF e IFP de un mismo dedo como para dos articulaciones MCF de diferentes dedos en la misma mano, muestran que esta forma de aplicación es efectiva y segura. En el caso de autorizarse la doble dosis simultánea en la práctica clínica habitual, se podría reducir el periodo de tratamiento, pero persistiría el problema del coste.

Las causas por las que fracasa una punción correctamente ejecutada no son bien conocidas, aunque probablemente influye la madurez y consistencia de las fibras de colágeno de la cuerda, cuya diversidad hemos podido apreciar en estudios con resonancia magnética. Cabe la posibilidad de que asegurando la colocación de la aguja en el centro de las cuerdas, mediante control ecográfico, pueda mejorarse la efectividad de la colagenasa. En la actualidad, estamos realizando con este objetivo un estudio comparativo entre la
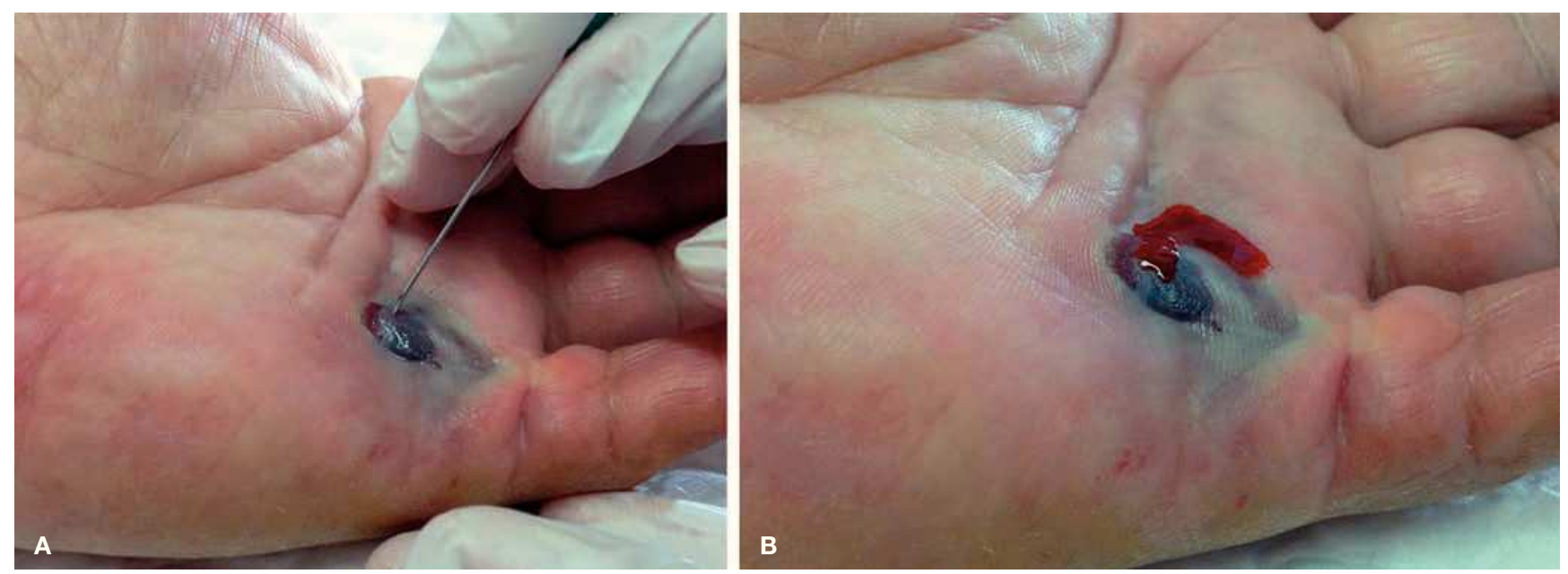

Figura 5 Flictena hemorrágica previa al estiramiento $(A)$, drenada mediante punción $(B)$. 
Tabla 1 Resultado clínico de publicaciones previas

\begin{tabular}{|c|c|c|c|c|c|c|c|c|}
\hline & $\begin{array}{l}\text { N. }{ }^{\circ} \\
\text { casos }\end{array}$ & $\begin{array}{l}\text { Media de } \\
\text { inyecciones }\end{array}$ & $\begin{array}{l}\text { Contractura } \\
\text { media global } \\
\text { inicio }\end{array}$ & $\begin{array}{l}\text { Contractura } \\
\text { media MTCF } \\
\text { inicio/final }\end{array}$ & $\begin{array}{l}\text { Contractura } \\
\text { media IFP } \\
\text { inicio/final }\end{array}$ & $\begin{array}{l}\text { Extensión } \\
\text { completa } \\
\text { global }\end{array}$ & $\begin{array}{l}\text { Extensión } \\
\text { completa } \\
\text { MTCF }\end{array}$ & $\begin{array}{l}\text { Extensión } \\
\text { completa } \\
\text { IFP }\end{array}$ \\
\hline Hurst $2009^{5}$ & 203 & 1,7 & $\begin{array}{l}149,1^{\circ} \pm \\
127,6^{\circ}\end{array}$ & - & - & $64 \%$ & $76,7 \%$ & $40 \%$ \\
\hline Gilpin $2010^{6}$ & 45 & 1,7 & $\begin{array}{l}174,7^{\circ} \pm \\
107,2^{\circ}\end{array}$ & - & - & $44,4 \%$ & $65 \%$ & $28 \%$ \\
\hline $\begin{array}{l}\text { Witthaut } \\
2013^{7}\end{array}$ & 879 & $1,2 \pm 0,5$ & $\begin{array}{l}135,1^{\circ} \pm \\
106^{\circ}\end{array}$ & - & - & $57 \%$ & $60 \%$ & $32 \%$ \\
\hline Peimer $2013^{11}$ & 1.080 & - & - & - & - & $58 \%$ & $\begin{array}{l}451 \\
\text { articul. }\end{array}$ & $\begin{array}{l}172 \\
\text { articul. }\end{array}$ \\
\hline $\begin{array}{l}\text { Martín } \\
\text { Ferrero } 2013^{3}\end{array}$ & 35 & 1 & - & $64^{\circ} / 4^{\circ}$ & $83,3^{\circ} / 15^{\circ}$ & - & - & - \\
\hline $\begin{array}{l}\text { Alberton } \\
2013^{10}\end{array}$ & 40 & 1 & - & $--/ 5,3^{\circ}$ & $--/ 6,8^{\circ}$ & $67,5 \%$ & - & - \\
\hline Peimer $2013^{9}$ & 629 & 1,08 & - & - & - & $67 \%$ & - & - \\
\hline $\begin{array}{l}\text { García Olea } \\
2014^{12}\end{array}$ & 126 & 1 & - & $41^{\circ}$ & $64^{\circ}$ & - & $80 \%$ & - \\
\hline
\end{tabular}

punción estándar y la ecodirigida. Otro grupo ${ }^{12}$ que ya ha efectuado punciones ecodirigidas no ha obtenido resultados superiores respecto a la punción normal.

Un aspecto del protocolo del tratamiento con colagenasa que puede ser modificado es el intervalo entre la inyección y la rotura de la cuerda. Si bien a partir de los primeros ensayos clínicos se ha venido recomendando dejar transcurrir 24 horas $^{5,6}$, el estudio de Manning ${ }^{18}$ muestra que pueden obtenerse similares buenos resultados efectuando el estiramiento al cabo de 48 horas. Esto amplía las opciones de organizar dentro de un Servicio la logística de tratamiento de los pacientes afectos de enfermedad de Dupuytren.

En nuestra serie no hemos observado ninguna reacción adversa grave, pero la frecuencia de desgarros cutáneos ha sido elevada. Si bien han tenido una evolución excelente con curas locales, constituyen un motivo de preocupación para el paciente y es imprescindible informar previamente de esta eventualidad. Entre las medidas que hemos puesto en práctica para minimizar estos desgarros cutáneos, pensamos que puede ser útil la evacuación de las flictenas hemorrágicas previamente al estiramiento, con objeto de reducir la tensión de la piel.

Demostrada la eficacia de la colagenasa en el tratamiento de la enfermedad del Dupuytren, es necesario efectuar un seguimiento a largo plazo de los casos tratados para valorar la tasa de recidivas y compararla con la obtenida mediante la cirugía abierta.

\section{Conclusión}

El uso de la colagenasa del Clostridium Histolyticum como tratamiento de la enfermedad de Dupuytren ofrece buenos resultados clínicos y un índice elevado de satisfacción en los pacientes a corto plazo. Los casos que mejor responden son los que presentan una cuerda pretendinosa con afectación exclusiva de la articulación MCF y las IFP que mejoran su contractura con la hiperflexión de la MCF.

\section{Conflicto de intereses}

Los autores declaran no tener ningún conflicto de intereses.

\section{Bibliografía}

1. Hurst L. Dupuytren's contracture. En: Green's operative hand surgery. Philadelphia: Elsevier Churchill Livingstone; 2011. p. 141-58.

2. Muñoz Peñin R, Lwoff N, Arandes Renú JM. Enfermedad de Dupuytren, una vista panorámica de nuestro entorno. Rev Iberoam Cir Mano. 2011;38:126-34.

3. Martín-Ferrero MA, Simón-Pérez C, Rodríguez-Mateos JI, GarcíaMedrano B, Hernández-Ramajo R, Brotat-García M. [Treatment of Dupuytren's disease using collagenase from Clostridium histolyticum]. Rev Esp Cir Ortop Traumatol. 2013;57:398-402.

4. Badalamente MA, Hurst LC. Efficacy and safety of injectable mixed collagenase subtypes in the treatment of Dupuytren's contracture. J Hand Surg Am. 2007;32:767-74.

5. Hurst LC, Badalamente MA, Hentz VR, Hotchkiss RN, Kaplan FT, Meals RA, et al.; CORD I Study Group. Injectable collagenase clostridium histolyticum for Dupuytren's contracture. N Engl J Med. 2009;361:968-79.

6. Gilpin D, Coleman S, Hall S, Houston A, Karrasch J, Jones N. Injectable collagenase Clostridium histolyticum: a new nonsurgical treatment for Dupuytren's disease. J Hand Surg Am. 2010;35:2027-38.

7. Witthaut J, Jones G, Skrepnik N, Kushner H, Houston A, Lindau TR. Efficacy and safety of collagenase clostridium histolyticum injection for Dupuytren contracture: short-term results from 2 open-label studies. J Hand Surg Am. 2013;38:2-11.

8. Badalamente MA, Hurst LC, Hentz VR. Collagen as a clinical target: nonoperative treatment of Dupuytren's disease. J Hand Surg Am. 2002;27:788-98. 
9. Peimer CA, Skodny P, Mackowiak JI. Collagenase clostridium histolyticum for dupuytren contracture: patterns of use and effectiveness in clinical practice. J Hand Surg Am. 2013;38: 2370-6.

10. Alberton F, Corain M, Garofano A, Pangallo L, Valore A, Zanella $\mathrm{V}$, et al. Efficacy and safety of collagenase Clostridium histolyticum injection for Dupuytren contracture: report of 40 cases. Musculoskelet Surg. 2014;98:225-32.

11. Peimer CA, Blazar P, Coleman S, Kaplan FT, Smith T, Tursi JP, et al. Dupuytren contracture recurrence following treatment with collagenase clostridium histolyticum (CORDLESS study): 3-year data. J Hand Surg Am. 2013;38:12-22.

12. García-Olea A, Dudley AF, Gimeno Andrade MD, Ocampos M, Fahandezh-Saddi H, Del Cerro M. Efectividad del tratamiento de la enfermedad de Dupuytren (ED) mediante fasciotomía enzimática con colagenasa. Estudio multicéntrico cohortes prospectivo. Rev Iberoam Cir Mano. 2014;42:97-106.

13. Gajendran VK, Hentz V, Kenney D, Curtin CM. Multiple collagenase injections are safe for treatment of Dupuytren's contractures. Orthopedics. 2014;37:e657-e660.

14. Chen NC, Shauver MJ, Chung KC. Cost-effectiveness of open partial fasciectomy, needle aponeurotomy, and collagenase injection for dupuytren contracture. J Hand Surg Am. 2011;36: 1826-34.
15. De Salas-Cansado M, Cuadros M, Del Cerro M, Arandes JM. Budget impact analysis in Spanish patients with Dupuytren's contracture: fasciectomy vs. collagenase Clostridium histolyticum. Chir Main. 2013;32:68-73.

16. Sanjuan Cerveró R, Franco Ferrando N, Poquet Jornet J. Use of resources and costs associated with the treatment of Dupuytren's contracture at an orthopedics and traumatology surgery department in Denia (Spain): collagenase clostridium hystolyticum versus subtotal fasciectomy. BMC Musculoskelet Disord. 2013;14:293.

17. Coleman S, Gilpin D, Kaplan FT, Houston A, Kaufman GJ, Cohen $B M$, et al. Efficacy and safety of concurrent collagenase clostridium histolyticum injections for multiple Dupuytren contractures. J Hand Surg Am. 2014;39:57-64.

18. Manning CJ, Delaney R, Hayton MJ. Efficacy and tolerability of day 2 manipulation and local anaesthesia after collagenase injection in patients with Dupuytren's contracture. J Hand Surg Eur Vol. 2014;39:466-71. 